\title{
Article
}

\section{The Injectivity Theorem on a Non-Compact Kähler Manifold}

\author{
Jingcao $\mathrm{Wu}$ (1)
}

check for updates

Citation: $\mathrm{Wu}, \mathrm{J}$. The Injectivity Theorem on a Non-Compact Kähler Manifold. Symmetry 2021, 13, 2222. https://doi.org/10.3390/sym13112222

Academic Editor: Roman Ger

Received: 20 October 2021

Accepted: 9 November 2021

Published: 20 November 2021

Publisher's Note: MDPI stays neutral with regard to jurisdictional claims in published maps and institutional affiliations.

Copyright: (c) 2021 by the author. Licensee MDPI, Basel, Switzerland. This article is an open access article distributed under the terms and conditions of the Creative Commons Attribution (CC BY) license (https:/ / creativecommons.org/licenses/by/ $4.0 /)$.
School of Mathematical Sciences, Shanghai University of Finance and Economics, Shanghai 200433, China; wujincao@mail.shufe.edu.cn

Abstract: In this paper, we establish an injectivity theorem on a weakly pseudoconvex Kähler manifold $X$ with negative sectional curvature. For this purpose, we develop the harmonic theory in this circumstance. The negative sectional curvature condition is usually satisfied by the manifolds with hyperbolicity, such as symmetric spaces, bounded symmetric domains in $\mathbb{C}^{n}$, hyperconvex bounded domains, and so on.

Keywords: non-compact Kähler manifold; Hodge decomposition; harmonic differential form; Hilbert space

MSC: Primary 32J25; Secondary 32Q15

\section{Introduction}

The injectivity theorem was first developed in [1,2] on a (compact) projective manifold $X$ for an ample line bundle $L$. Then, it is generalized by a series of articles, such as [3-9], eventually to a compact Kähler manifold $X$ with pseudo-effective line bundle $L$. After that, it is natural to seek the similar result on a non-compact manifold. To my best acknowledgement, there are only a few results, such as $[10,11]$, in this aspect.

In this paper, we are interested in the manifolds with convexity. More precisely, let $(X, \omega)$ be a weakly pseudoconvex Kähler manifold. By this, we mean a Kähler manifold $X$ such that there exists a smooth plurisubharmonic exhaustion function $\psi$ on $X(\psi$ is said to be an exhaustion if for every $c>0$ the upperlevel set $X_{c}=\psi^{-1}(c)$ is relatively compact, i.e., $\psi(z)$ tends to $+\infty$ when $z$ is taken outside larger and larger compact subsets of $X$ ). We prove that

Theorem 1. Let $(X, \omega)$ be a weakly pseudoconvex Kähler manifold such that the sectional curvature

$$
\text { sec } \leqslant-K(\text { see Definition } 3)
$$

for some positive constant $K$. Let $\left(L, \varphi_{L}\right)$ and $\left(H, \varphi_{H}\right)$ be two (singular) Hermitian line bundles on $X$. Assume the following conditions:

1. There exists a closed subvariety $Z$ on $X$ such that $\varphi_{L}$ and $\varphi_{H}$ are smooth on $X \backslash Z$;

2. $i \Theta_{L, \varphi_{L}} \geqslant 0$ and $i \Theta_{H, \varphi_{H}} \geqslant 0$ on $X$;

3. $i \Theta_{L, \varphi_{L}} \geqslant \delta i \Theta_{H, \varphi_{H}}$ for some positive number $\delta$.

For a (non-zero) section s of $H$ with $\sup _{X}|s|^{2} e^{-\varphi_{H}}<\infty$, the multiplication map induced by the tensor product with $\mathrm{s}$

$$
\Phi: H^{q}\left(X, K_{X} \otimes L \otimes \mathscr{I}\left(\varphi_{L}\right)\right) \rightarrow H^{q}\left(X, K_{X} \otimes L \otimes H \otimes \mathscr{I}\left(\varphi_{L}+\varphi_{H}\right)\right)
$$

is (well-defined and) injective for any $q \geqslant 0$.

Remark 1. The assumption (1) can be immediately removed if Demailly's approximation technique [12] is valid in this situation. However, it seems to me that the compactness of the base 
manifold is of crucial importance in his original proof. Thus, it is hard to directly apply his argument here. We are interested to know whether such an approximation exists on a non-compact manifold.

We will recall the definition of singular metric and multiplier ideal sheaf $\mathscr{I}\left(\varphi_{L}\right)$ in Section 2, and the elementary properties of manifolds with negative sectional curvature in Section 3.

Theorem 1 implies the following $L^{2}$-extension theorem concerning the subvariety that is not necessary to be reduced. Such type of extension problem was studied in [10] before.

Corollary 1. Let $(X, \omega)$ be a weakly pseudoconvex Kähler manifold such that

$$
\sec \leqslant-K
$$

for some positive constant $K$. Let $\left(L, \varphi_{L}\right)$ be a (singular) Hermitian line bundle on $X$, and let $\varphi$ be a quasi-plurisubharmonic function on X. Assume the following conditions:

1. There exists a closed subvariety $Z$ on $X$ such that $\varphi_{L}$ is smooth on $X \backslash Z$;

2. $i \Theta_{L, \varphi_{L}} \geqslant 0$;

3. $i \Theta_{L, \varphi_{L}}+(1+\delta) i \partial \bar{\partial} \varphi \geqslant 0$ for all non-negative number $\delta \in[0, \varepsilon)$ with $0<\varepsilon \ll 1$.

Then, the natural morphism

$$
H^{0}\left(X, K_{X} \otimes L \otimes \mathscr{I}\left(\varphi_{L}\right)\right) \rightarrow H^{0}\left(X, K_{X} \otimes L \otimes \mathscr{I}\left(\varphi_{L}\right) / \mathscr{I}\left(\varphi_{L}+\varphi\right)\right)
$$

is surjective.

Remark 2. If $\varphi_{L}$ is smooth, we have $\mathscr{I}\left(\varphi_{L}\right)=\mathcal{O}_{X}$ and

$$
\mathscr{I}\left(\varphi_{L}\right) / \mathscr{I}\left(\varphi_{L}+\varphi\right)=\mathcal{O}_{X} / \mathscr{I}(\varphi)=: \mathcal{O}_{Y}
$$

where $Y$ is the subvariety defined by the ideal sheaf $\mathscr{I}(\varphi)$. In particular, $Y$ is not necessary to be reduced. Then, the surjectivity statement can interpret an extension theorem for holomorphic sections, with respect to the restriction morphism

$$
H^{0}\left(X, K_{X} \otimes L\right) \rightarrow H^{0}\left(Y,\left.\left(K_{X} \otimes L\right)\right|_{Y}\right)
$$

In order to prove Theorem 1, we improve the $L^{2}$-Hodge theory introduced in [13], such that it is suitable for the forms taking value in a line bundle. The crucial thing is the Hodge decomposition $[14,15]$ on a non-compact manifold. Since the base manifold has negative sectional curvature, it is Kähler hyperbolic by [13]. We then apply the Kähler hyperbolicity to establish the Hodge decomposition. We leave all the details in the text.

Remark 3. The Kähler hyperbolic manifold was deeply studied in [13]. In particular, Ref. [13] provides many examples for Kähler hyperbolic manifolds, such as symmetric spaces, bounded symmetric domains in $\mathbb{C}^{n}$, hyperconvex bounded domains, and so on. Certainly, Theorem 1 is valid on these manifolds.

Remark 4. All the results are still valid if $L$ is twisted by a Nakano semi-positive [16] vector bundle E. The proof involves nothing new hence we omit it.

The plan of this paper is as follows: we will first recall the background materials in Section 2. The Kähler hyperbolicity is discussed in Section 3. Then, we discuss the Hodge decomposition on a non-compact manifold in Section 4. In Section 5, we prove the injectivity theorem and the extension theorem. 


\section{Preliminarily}

\subsection{Singular Metric}

Recall that a smooth Hermitian metric $h$ on a line bundle $L$ is given in any trivialization $\theta:\left.L\right|_{U} \simeq U \times \mathbb{C}$ by

$$
\|\alpha\|_{h}^{2}=|\theta(\alpha)|^{2} e^{-2 \varphi(x)}, x \in U, \alpha \in L_{x}
$$

where $\varphi \in C^{\infty}(U)$ is an arbitrary function, called the weight of the metric with respect to the trivialization $\theta$. Then, the singular Hermitian metric is defined in [16] as follows:

Definition 1 (Singular metric). A singular Hermitian metric $h$ on a line bundle $L$ is given in any trivialization $\theta:\left.L\right|_{U} \simeq U \times \mathbb{C}$ by

$$
\|\alpha\|_{h}^{2}=|\theta(\alpha)|^{2} e^{-2 \varphi(x)}, x \in U, \alpha \in L_{x}
$$

where $\varphi \in L^{1}(U)$ is an arbitrary function, called the weight of the metric with respect to the trivialization $\theta$.

Sometimes, we will directly say that $\varphi$ is a (singular) metric on $L$ if nothing is confused.

\subsection{Multiplier Ideal Sheaf}

The multiplier ideal sheaf is an important tool in modern complex geometry, which was originally introduced in $[16,17]$.

Definition 2 (Multiplier ideal sheaf). Let $L$ be a line bundle. Let $\varphi$ be a singular metric on $L$ such that $i \Theta_{L, \varphi} \geqslant \gamma$ for a smooth real $(1,1)$-form $\gamma$ on $X$. Then, the multiplier ideal sheaf is defined as

$$
\begin{aligned}
\mathscr{I}(\varphi)_{x}:= & \left\{f \in \mathcal{O}_{X, x} ; \text { there exists a small neighborhood } U_{x}\right. \text { of } \\
& \left.x \text { such that } \int_{U_{x}}|f|^{2} e^{-2 \varphi}<\infty\right\} .
\end{aligned}
$$

Note that $X$ is non-compact, and $f \in \Gamma(X, \mathscr{I}(\varphi))$ in general will not imply that

$$
\int_{X}|f|^{2} e^{-2 \varphi}<\infty
$$

However, when $X$ is furthermore assumed to be weakly pseudoconvex, we could substitute $\varphi+\chi \circ \psi$ for $\varphi$. Here, $\chi$ is a convex increasing function of arbitrary fast growth at infinity and $\psi$ is the smooth plurisubharmonic exhaustion function provided by the weak pseudoconvexity of $X$. This factor can be used to ensure the convergence of integrals at infinity. Moreover, we have

$$
\mathscr{I}(\varphi+\chi \circ \psi)=\mathscr{I}(\varphi)
$$

and $i \Theta_{L, \varphi+\chi \circ \psi} \geqslant \gamma$. Therefore, we can always assume without loss of generality that, for every $f \in \Gamma(X, \mathscr{I}(\varphi))$,

$$
\int_{X}|f|^{2} e^{-2 \varphi}<\infty
$$

\section{The Kähler Manifold with Negative Curvature}

\subsection{Negative Curvature}

Firstly, let us recall the definition for a manifold with negative sectional curvature.

Definition 3. Let $(X, \omega)$ be a Kähler manifold. Let $\left(\Theta_{i j \alpha} \bar{\beta}\right)_{1 \leqslant i, j, \alpha, \beta \leqslant n}$ be the curvature associated with $\omega$. Then, $X$ is said to have negative sectional curvature, if there exists a positive constant $K$ such that, for any non-zero complex vector $\xi=\left(\xi_{1}, \ldots, \xi_{n}\right)$,

$$
\sum i \Theta_{i j \alpha \bar{\beta}} \bar{\zeta}_{i} \bar{\xi}_{j} \xi_{\alpha} \bar{\xi}_{\beta} \leqslant-K\|\xi\|^{2}
$$


It is denoted by $\mathrm{sec} \leqslant-K$.

A complete Kähler manifold with negative sectional curvature will be Kähler hyperbolic (see Proposition 1). The Kähler hyperbolicity was first introduced in [13] for a compact Kähler manifold. However, there is no obstacle to extend it to the non-compact case. Firstly, let us recall the $d$-boundedness of a differential form.

Definition 4. Let $\alpha$ be a differential form on $X$. Let $\pi: \tilde{X} \rightarrow X$ be the universal covering of $X$. Then,

(i) $\alpha$ is called bounded (with respect to $\omega$ ) if the $L^{\infty}$-norm of $\alpha$ is finite,

$$
\|\alpha\|_{L^{\infty}}:=\sup _{x \in X}|\alpha|_{\omega}<\infty .
$$

Here, $|\alpha|_{\omega}$ is the pointwise norm induced by $\omega$.

(ii) $\alpha$ is called $d$-bounded if there exists a differential form $\beta$ on $X$ such that $\alpha=d \beta$ and $\|\beta\|_{L^{\infty}}<\infty$.

(iii) $\alpha$ is called $\tilde{d}$-bounded if $\pi^{*} \alpha$ is $d$-bounded on $\tilde{X}$.

Remark 5. When $X$ is compact, these notions bring nothing new. When $X$ is non-compact, it is easy to verify that d-boundedness implies $\tilde{d}$-boundedness, whereas there is no direct relationship between boundedness and d-bounxdedness.

The Kähler hyperbolic manifold is then defined as

Definition 5. A Kähler manifold $(X, \omega)$ is called Kähler hyperbolic if $\omega$ is $\tilde{d}$-bounded.

We list some functionality property of the Kähler hyperbolicity here. They are almost obvious, and one could refer to [13] for more details.

\section{Proposition 1.}

(i) Let X be a Kähler hyperbolic manifold. Then, every complex submanifold of $X$ is still Kähler hyperbolic. In fact, if $Y$ is a complex manifold which admits a finite morphism $Y \rightarrow X$, then $Y$ is Kähler hyperbolic.

(ii) Cartesian product of Kähler hyperbolic manifolds is Kähler hyperbolic.

(iii) A complete Kähler manifold $(X, \omega)$ with negative sectional curvature must be Kähler hyperbolic. This fact was pointed out in [13], whose proof can be found in [18]. More precisely, if sec $\leqslant-K$, there exists a 1-form $\eta$ on $\tilde{X}$ such that $\pi^{*} \omega=d \eta$ and

$$
\|\eta\|_{L^{\infty}} \leqslant K^{-\frac{1}{2}}
$$

\subsection{Notations and Conventions}

We make a brief introduction for the basic notations and conventions in Kähler geometry to finish this section. We recommend readers to see [15] for a sophisticated comprehension.

Let $(X, \omega)$ be a Kähler manifold of dimension $n$, and let $(L, \varphi)$ be a holomorphic line bundle on $X$ endowed with a smooth metric $\varphi$. The standard operators, such as $\bar{\partial}$, * as well as $L, \Lambda$, etc., in Kähler geometry are defined locally and thus make sense with or without the compactness or completeness assumptions. For an $m$-form $\theta$, we define $e(\theta):=\theta \wedge$. Let $D=\partial_{\varphi}+\bar{\partial}$ be the Chern connection on $L$ associated with $\varphi$. Moreover, for an $L$-valued $k$-form $\alpha$, we define the operators $D^{*} \alpha:=(-1)^{n+n k+1} * D * \alpha, \bar{\partial}^{*} \alpha:=(-1)^{n+n k+1} * \partial_{\varphi} * \alpha$, $\partial_{\varphi}^{*} \alpha:=(-1)^{n+n k+1} * \bar{\partial} * \alpha$ and $e(\theta)^{*} \alpha:=(-1)^{m(k+1)} * e(\theta) * \alpha$.

Let $A^{p, q}(X, L)$ be the space of all the smooth $L$-valued $(p, q)$-forms on $X$. The pointwise inner product $\left\langle\cdot, \cdot>_{\omega, \varphi}\right.$ on $A^{p, q}(X, L)$ is defined by the equation:

$$
<\alpha, \beta>{ }_{\omega, \varphi} d V_{\omega}:=\alpha \wedge * \bar{\beta} e^{-2 \varphi}
$$


for $\alpha, \beta \in A^{p, q}(X, L)$. The pointwise norm $|\cdot| \omega, \varphi$ is then induced by $<\cdot, \cdot>_{\omega, \varphi}$. The $L^{2}$-inner product is defined by

$$
(\alpha, \beta)_{\omega, \varphi}:=\int_{X}<\alpha, \beta>_{\omega, \varphi} d V_{\omega}
$$

for $\alpha, \beta \in A^{p, q}(X, L)$, and the norm $\|\cdot\|_{\omega, \varphi}$ is induced by $(\cdot, \cdot)_{\omega, \varphi}$.

Let $L_{(2)}^{p, q}(X, L)$ be the space of all the $L$-valued (not necessary to be smooth) $(p, q)$-forms with bounded $L^{2}$-norm on $X$, and it equipped with $(\cdot, \cdot)_{\omega, \varphi}$ becomes a Hilbert space. The operators $D^{*}, \bar{\partial}^{*}$, and $\partial_{\varphi}^{*}$ are then the adjoint operators of $D, \bar{\partial}$, and $\partial_{\varphi}$ with respect to $(\cdot, \cdot)_{\omega, \varphi}$ if $X$ is compact. However, when $X$ is non-compact, the situation would be much more complicated. We will deal with it in the next section.

\section{The Hodge Decomposition}

The Hodge decomposition is the ingredient to study the geometry of a compact Kähler manifold. One can consult [14,15] for a complete survey. In this section, we will discuss the Hodge decomposition on a non-compact manifold. Let $(X, \omega)$ be a complete Kähler manifold of dimension $n$ with negative sectional curvature, and let $(L, \varphi)$ be a holomorphic line bundle on $X$ endowed with a smooth metric $\varphi$.

\subsection{Elementary Materials}

We collect from [13] some basic properties concerning the Hodge decomposition here. Remember that the adjoint relationship between $\bar{\partial}$ and $\bar{\partial}^{*}$ in general fails when $X$ is non-compact. In fact, the compactness becomes important when one takes an integral. However, since $X$ is complete here, we still have the Stokes formula as follows:

Lemma 1. Let $\eta$ be an $L$-valued $L^{1}$-bounded $(2 n-1)$-form on $X$, i.e.,

$$
\|\eta\|_{L^{1}}:=\int_{X}|\eta|_{\omega} d V_{\omega}<\infty
$$

such that $d \eta$ is also $L^{1}$-bounded. Then,

$$
\int_{X} d \eta=0
$$

Essentially, this lemma is not a surprise after applying the cut-off function to reduce it to the case that $\eta$ has the compact support, while the existence of such a cut-off function is guaranteed by the completeness of $\omega$. For example, we could use the geodesic distance $\delta_{\omega}$ to construct a function $a_{\varepsilon}$ on $X$ for every $\varepsilon>0$ satisfying the following conditions:

1. $a_{\varepsilon}$ is smooth and takes values in the interval $[0,1]$ with compact support;

2. The subset $a_{\varepsilon}^{-1}(1) \subset X$ exhausts $X$ as $\varepsilon$ tends zero, and

3. $\left\|d a_{\varepsilon}\right\|_{L^{\infty}} \leqslant \varepsilon$.

Now the proof of Lemma 1 is elementary, and we omit it here. With the help of Lemma 1, most of the canonical identities on compact Kähler manifold extend into this situation. Remember that the Laplacian operators are defined as

$$
\begin{aligned}
& \square=D D^{*}+D^{*} D, \square_{\bar{\partial}}=\bar{\partial} \bar{\partial}^{*}+\bar{\partial}^{*} \bar{\partial} \text { and } \\
& \square_{\partial}=\partial_{\varphi} \partial_{\varphi}^{*}+\partial_{\varphi}^{*} \partial_{\varphi}, \text { respectively. }
\end{aligned}
$$

Proposition 2. Let $\alpha$ be an L-valued $L^{2}$-bounded form on X. Then, 
1. Integral identities.

$$
\begin{aligned}
& (\square \alpha, \alpha)_{\omega, \varphi}=(D \alpha, D \alpha)_{\omega, \varphi}+\left(D^{*} \alpha, D^{*} \alpha\right)_{\omega, \varphi} \\
& \left(\square_{\bar{\partial}} \alpha, \alpha\right)_{\omega, \varphi}=(\bar{\partial} \alpha, \bar{\partial} \alpha)_{\omega, \varphi}+\left(\bar{\partial}^{*} \alpha, \bar{\partial}^{*} \alpha\right)_{\omega, \varphi} \text { and } \\
& \left(\square_{\partial} \alpha, \alpha\right)_{\omega, \varphi}=\left(\partial_{\varphi} \alpha, \partial_{\varphi} \alpha\right)_{\omega, \varphi}+\left(\partial_{\varphi}^{*} \alpha, \partial_{\varphi}^{*} \alpha\right)_{\omega, \varphi} .
\end{aligned}
$$

2. Bochner-Kodaira-Nakano identity.

$$
\square_{\bar{\partial}}=\square_{\partial}+\left[i \Theta_{L, \varphi}, \Lambda\right] .
$$

In particular, 1. and 2. together give that

$$
\begin{aligned}
& (\bar{\partial} \alpha, \bar{\partial} \alpha)_{\omega, \varphi}+\left(\bar{\partial}^{*} \alpha, \bar{\partial}^{*} \alpha\right)_{\omega, \varphi} \\
= & \left(\partial_{\varphi} \alpha, \partial_{\varphi} \alpha\right)_{\omega, \varphi}+\left(\partial_{\varphi}^{*} \alpha, \partial_{\varphi}^{*} \alpha\right)_{\omega, \varphi}+\left(\left[i \Theta_{L, \varphi}, \Lambda\right] \alpha, \alpha\right)_{\omega, \varphi}
\end{aligned}
$$

Proof. We only prove that

$$
(\square \alpha, \alpha)_{\omega, \varphi}=(D \alpha, D \alpha)_{\omega, \varphi}+\left(D^{*} \alpha, D^{*} \alpha\right)_{\omega, \varphi} .
$$

Recall that, for any differential forms $\alpha, \beta$ with proper degree, we always have

$$
D \alpha \wedge * \bar{\beta} e^{-2 \varphi}-\alpha \wedge * \overline{D^{*} \beta} e^{-2 \varphi}= \pm d\left(\alpha \wedge * \bar{\beta} e^{-2 \varphi}\right),
$$

where the sign on the right-hand side is determined by the degree of $\alpha$. Therefore,

$$
\begin{aligned}
(\square \alpha, \alpha)_{\omega, \varphi}= & \lim _{\varepsilon \rightarrow 0}\left(\square \alpha, a_{\varepsilon} \alpha\right)_{\omega, \varphi} \\
= & \lim _{\varepsilon \rightarrow 0}\left(\left(D \alpha, D\left(a_{\varepsilon} \alpha\right)\right)_{\omega, \varphi}+\left(D^{*} \alpha, D^{*}\left(a_{\varepsilon} \alpha\right)\right)_{\omega, \varphi}\right. \\
& \pm \int_{X} d\left(D^{*} \alpha \wedge * a_{\varepsilon} \bar{\alpha} e^{-2 \varphi}\right) \pm \int_{X} d\left(* \overline{D \alpha} \wedge a_{\varepsilon} \alpha e^{-2 \varphi}\right) \\
= & (D \alpha, D \alpha)_{\omega, \varphi}+\left(D^{*} \alpha, D^{*} \alpha\right)_{\omega, \varphi}+\lim _{\varepsilon \rightarrow 0}\left(D \alpha, e\left(d a_{\varepsilon}\right) \alpha\right)_{\omega, \varphi} \\
& +\lim _{\varepsilon \rightarrow 0}\left(D^{*} \alpha, e\left(d a_{\varepsilon}\right)^{*} \alpha\right)_{\omega, \varphi} .
\end{aligned}
$$

We apply Lemma 1 to obtain the third equality. Obviously,

$$
\begin{aligned}
I_{\mathcal{\varepsilon}} & :=\left|\left(D \alpha, e\left(d a_{\varepsilon}\right) \alpha\right)_{\omega, \varphi}\right|+\left|\left(D^{*} \alpha, e\left(d a_{\varepsilon}\right)^{*} \alpha\right)_{\omega, \varphi}\right| \\
& \leqslant \int_{X}\left|d a_{\varepsilon}\right|_{\omega}|\alpha|_{\omega, \varphi}\left(|D \alpha|_{\omega, \varphi}+\left|D^{*} \alpha\right|_{\omega, \varphi}\right) .
\end{aligned}
$$

Then, we choose $a_{\varepsilon}$ such that $\left|d a_{\varepsilon}\right|_{\omega}^{2} \leqslant \varepsilon a_{\varepsilon}$ on $X$ and estimate $I_{\mathcal{\varepsilon}}$ by Schwarz inequality. This yields

$$
I_{\varepsilon} \leqslant 2 \varepsilon\|\alpha\|_{\omega, \varphi}\left(\int_{X}\left|a_{\varepsilon}\right|\left(|D \alpha|_{\omega, \varphi}^{2}+\left|D^{*} \alpha\right|_{\omega, \varphi}^{2}\right)\right)^{1 / 2} .
$$

Hence, $I_{\varepsilon} \rightarrow 0$ as $\varepsilon$ tends to zero. As a result, we obtain the desired equality.

The other identities are similar.

There are various quick consequences of this proposition. For example, $\alpha$ is $\square$ harmonic, i.e., $\square \alpha=0$, if and only if $D \alpha=0$ and $D^{*} \alpha=0$. The similar conclusion holds for the operators $\square_{\bar{\jmath}}$ and $\square_{\partial}$.

Moreover, with Lemma 1 and Proposition 2, one concludes that the $L^{2}$-space $L_{(2)}^{k}(X, L)$ of the $L$-valued $k$-forms on $X$ admits Hodge decomposition as follows: 
Definition 6 (Hodge decomposition, I). For the $L^{2}$-space $L_{(2)}^{k}(X, L)$, we have the following orthogonal decomposition:

$$
L_{(2)}^{k}(X, L)=\overline{\operatorname{Im} D} \oplus \mathcal{H}^{k}(L) \oplus \overline{\operatorname{Im} D^{*}}
$$

where

$$
\begin{gathered}
\operatorname{Im} D=\operatorname{Im}\left(D: L_{(2)}^{k-1}(X, L) \rightarrow L_{(2)}^{k}(X, L)\right), \\
\mathcal{H}^{k}(L)=\left\{\alpha \in L_{(2)}^{k}(X, L) ; D \alpha=0, D^{*} \alpha=0\right\},
\end{gathered}
$$

and

$$
\operatorname{Im} D^{*}=\operatorname{Im}\left(D^{*}: L_{(2)}^{k+1}(X, L) \rightarrow L_{(2)}^{k}(X, L)\right) .
$$

Similarly, for the $L^{2}$-space $L_{(2)}^{p, q}(X, L)$ of the $L$-valued $(p, q)$-forms, we have

Definition 7 (Hodge decomposition, II).

$$
L_{(2)}^{p, q}(X, L)=\overline{\operatorname{Im} \bar{\partial}} \oplus \mathcal{H}^{p, q}(L) \oplus \overline{\operatorname{Im} \bar{\partial}^{*}}
$$

where

$$
\begin{aligned}
\operatorname{Im} \bar{\partial} & =\operatorname{Im}\left(\bar{\partial}: L_{(2)}^{p, q-1}(X, L) \rightarrow L_{(2)}^{p, q}(X, L)\right), \\
\mathcal{H}^{p, q}(L) & =\left\{\alpha \in L_{(2)}^{p, q}(X, L) ; \bar{\partial} \alpha=0, \bar{\partial}^{*} \alpha=0\right\},
\end{aligned}
$$

and

$$
\operatorname{Im} \bar{\partial}^{*}=\operatorname{Im}\left(\bar{\partial}^{*}: L_{(2)}^{p, q+1}(X, L) \rightarrow L_{(2)}^{p, q}(X, L)\right) .
$$

\subsection{Lower Bound on the Spectrum}

In this section, we will show that $\operatorname{Im} D$ and $\operatorname{Im} D^{*}$ in the decomposition (1), $\operatorname{Im} \bar{\partial}$, and $\operatorname{Im} \bar{\partial}^{*}$ in the decomposition (2) are actually closed, in which the negative sectional curvature really comes into effect. Remembering that $(X, \omega)$ is Kähler hyperbolic by Proposition 1, we have $\pi^{*} \omega=d \eta$, where $\pi: \tilde{X} \rightarrow X$ is the universal covering and $\eta$ is a bounded form on $\tilde{X}$.

Let $\tilde{\omega}=\pi^{*} \omega, \tilde{L}=\pi^{*} L$ and $\Phi=\pi^{*} \varphi$. The $L^{2}$-spaces

$$
\left(L_{(2)}^{k}(\tilde{X}, \tilde{L}),(\cdot, \cdot)_{\tilde{\omega}, \Phi}\right) \text { and }\left(L_{(2)}^{p, q}(\tilde{X}, \tilde{L}),(\cdot, \cdot)_{\tilde{\omega}, \Phi}\right)
$$

and the related subsets such as $\operatorname{Im} D, \operatorname{Im} D^{*}$ are defined in an obvious way.

Proposition 3. Every $\alpha \in L_{(2)}^{k}(\tilde{X}, \tilde{L})$ with $k \neq m$ satisfies that

$$
(\square \alpha, \alpha)_{\tilde{\omega}, \Phi} \geqslant \lambda^{2}(\alpha, \alpha)_{\tilde{\omega}, \Phi},
$$

where $\lambda$ is a strictly positive constant which depends only on $k, m=\operatorname{dim} \tilde{X}$ and $\|\eta\|_{L^{\infty}}$. Furthermore, when $k=m$ inequality (3) is satisfied by $\alpha \in L_{(2)}^{m}(\tilde{X}, \tilde{L})$, which is orthogonal to $\mathcal{H}^{m}(\tilde{L})$.

Proof. When $k \neq m$, inequality (3) was proved in [19]. According to Proposition 2, it shows that the $D$-closed $\tilde{L}$-valued $k$-form $\alpha(k \neq m)$ satisfies

$$
\|\alpha\|_{\tilde{\omega}, \Phi}^{2} \leqslant \lambda^{-2}\left\|D^{*} \alpha\right\|_{\tilde{\omega}, \Phi}^{2}
$$


and the $D^{*}$-closed $\tilde{L}$-valued $k$-form $\alpha(k \neq m)$ satisfies

$$
\|\alpha\|_{\tilde{\omega}, \Phi}^{2} \leqslant \lambda^{-2}\|D \alpha\|_{\tilde{\omega}, \Phi}^{2}
$$

Therefore, $\operatorname{Im} D$ as well as $\operatorname{Im} D^{*}$ is itself closed.

In particular, applying the conclusion above to the $\tilde{L}$-valued $D^{*}$-closed $(m-1)$-form and $\tilde{L}$-valued $D$-closed $(m+1)$-form respectively, we obtain that

$$
\operatorname{Im} D=\operatorname{Im}\left(D: L_{(2)}^{m-1}(\tilde{X}, \tilde{L}) \rightarrow L_{(2)}^{m}(\tilde{X}, \tilde{L})\right)
$$

and

$$
\operatorname{Im} D^{*}=\operatorname{Im}\left(D^{*}: L_{(2)}^{m+1}(\tilde{X}, \tilde{L}) \rightarrow L_{(2)}^{m}(\tilde{X}, \tilde{L})\right)
$$

are both closed. Therefore, Hodge decomposition is improved as

$$
L_{(2)}^{m}(\tilde{X}, \tilde{L})=\operatorname{Im} D \oplus \mathcal{H}^{m}(\tilde{L}) \oplus \operatorname{Im} D^{*}
$$

for $k=m$.

Now we are able to prove the proposition for the form $\alpha$ of degree $m$ orthogonal to $\mathcal{H}^{m}(\tilde{L})$. We have $\alpha=D \beta+D^{*} \gamma$, where $D \beta$ is orthogonal to $D^{*} \gamma$, and the $L^{2}$-forms $\beta$ and $\gamma$ of degrees $m-1$ and $m+1$ correspondingly satisfy $D^{*} \beta=0, D \gamma=0$. This implies

$$
(\alpha, \alpha)_{\tilde{\omega}, \Phi}=(D \beta, D \beta)_{\tilde{\omega}, \Phi}+\left(D^{*} \gamma, D^{*} \gamma\right)_{\tilde{\omega}, \Phi}=(\square \beta, \beta)_{\tilde{\omega}, \Phi}+(\square \gamma, \gamma)_{\tilde{\omega}, \Phi},
$$

as well as $\square \beta=D^{*} \alpha$ and $\square \gamma=D \alpha$. On the other hand, apply inequality (3) to $\beta$ and $\gamma$ yields, as a consequence of Schwarz inequality that

$$
(\square \beta, \beta)_{\tilde{\omega}, \Phi} \leqslant \lambda^{-2}(\square \beta, \square \beta)_{\tilde{\omega}, \Phi}
$$

and

$$
(\square \gamma, \gamma)_{\tilde{\omega}, \Phi} \leqslant \lambda^{-2}(\square \gamma, \square \gamma)_{\tilde{\omega}, \Phi}
$$

Thus,

$$
\begin{aligned}
(\alpha, \alpha)_{\tilde{\omega}, \Phi} & \leqslant \lambda^{-2}\left(\left(D^{*} \alpha, D^{*} \alpha\right)_{\tilde{\omega}, \Phi}+(D \alpha, D \alpha)_{\tilde{\omega}, \Phi}\right) \\
& =\lambda^{-2}(\square \alpha, \alpha)_{\tilde{\omega}, \Phi} .
\end{aligned}
$$

If the curvature of $L$ is bounded from below, we will have a similar estimate for $\square_{\bar{\jmath}}$.

Corollary 2. Assume that $i \Theta_{L, \varphi} \geqslant k \omega$ for a constant $k$. Then, $\alpha \in L_{(2)}^{p, q}(\tilde{X}, \tilde{L})$ with $p+q \neq m$ satisfies that

$$
\left(\square_{\tilde{\partial}} \alpha, \alpha\right)_{\tilde{\omega}, \Phi} \geqslant \frac{\lambda^{2}+k(p+q-m)}{2}(\alpha, \alpha)_{\tilde{\omega}, \Phi,}
$$

where $\lambda$ is the same constant in (3). Furthermore, when $p+q=m$, inequality (4) is satisfied by $\alpha \in L_{(2)}^{p, q}(\tilde{X}, \tilde{L})$, which is orthogonal to $\mathcal{H}^{p, q}(\tilde{L})$.

Proof. Since $\pi$ is locally isometric, $i_{\tilde{L}, \Phi} \geqslant k \tilde{\omega}$. By Proposition 2, we have

$$
\square=\square_{\partial}+\square_{\bar{\jmath}}=2 \square_{\bar{\jmath}}-\left[i \Theta_{\tilde{L}, \Phi}, \Lambda\right] .
$$

Therefore, when $p+q \neq m$,

$$
\begin{aligned}
\left(\square_{\tilde{\partial}} \alpha, \alpha\right)_{\tilde{\omega}, \Phi} & =\frac{1}{2}\left((\square \alpha, \alpha)_{\tilde{\omega}, \Phi}+\left(\left[i \Theta_{\tilde{L}, \Phi}, \Lambda\right] \alpha, \alpha\right)_{\tilde{\omega}, \Phi}\right) \\
& \geqslant \frac{\lambda^{2}+k(p+q-m)}{2}(\alpha, \alpha)_{\tilde{\omega}, \Phi} .
\end{aligned}
$$


The last inequality is due to Proposition 3 and elementary computation. Then, we apply the same argument as Proposition 3 to obtain the desired conclusion for $p+q=m$. The proof is complete.

In particular, if $\lambda^{2}+k(p+q-m)>0$ and $p+q \neq m$, the $\bar{\partial}$-closed $\tilde{L}$-valued $(p, q)$-form $\alpha$ satisfies

$$
\|\alpha\|_{\tilde{\omega}, \Phi}^{2} \leqslant \operatorname{const}_{k, m, \eta}\left\|\bar{\partial}^{*} \alpha\right\|_{\tilde{\omega}, \Phi}^{2}
$$

and the $\bar{\partial}^{*}$-closed $\tilde{L}$-valued $(p, q)$-form $\alpha$ satisfies

$$
\|\alpha\|_{\tilde{\omega}, \Phi}^{2} \leqslant \text { const }_{k, m, \eta}\|\bar{\partial} \alpha\|_{\tilde{\omega}, \Phi}^{2} .
$$

Here, const $t_{k, m, \eta}$ is a positive constant. Therefore, $\operatorname{Im} \bar{\partial}$ as well as $\operatorname{Im} \bar{\partial}^{*}$ is itself closed.

Therefore, we see that, when $\lambda^{2}+k(p+q-m)>0$ and $p+q \neq m-1$ or $m+1$, Hodge decomposition (2) can be improved on $\tilde{X}$ as:

$$
L_{(2)}^{p, q}(\tilde{X}, \tilde{L})=\operatorname{Im} \bar{\partial} \oplus \mathcal{H}^{p, q}(\tilde{L}) \oplus \operatorname{Im}^{*} .
$$

Thus, we have Hodge's theorem on $X$ as follows.

Proposition 4. Assuming that $i \Theta_{L, \varphi} \geqslant-\varepsilon \omega$ for some $\varepsilon$ small enough, then

$$
\mathcal{H}^{p, q}(L) \simeq \frac{\operatorname{Ker}\left(\bar{\partial}: L_{(2)}^{p, q}(X, L) \rightarrow L_{(2)}^{p, q+1}(X, L)\right)}{\operatorname{Im}\left(\bar{\partial}: L_{(2)}^{p, q-1}(X, L) \rightarrow L_{(2)}^{p, q}(X, L)\right)} .
$$

Proof. Firstly, we claim that

$$
\operatorname{Im} \bar{\partial}_{1}=\operatorname{Im}\left(\bar{\partial}: L_{(2)}^{p, q}(X, L) \rightarrow L_{(2)}^{p, q+1}(X, L)\right)
$$

is closed provided that

$$
\operatorname{Im} \bar{\partial}_{2}=\operatorname{Im}\left(\bar{\partial}: L_{(2)}^{p, q}(\tilde{X}, \tilde{L}) \rightarrow L_{(2)}^{p, q+1}(\tilde{X}, \tilde{L})\right)
$$

is closed. In order to prove this claim, let us review the relationship between the $L^{2}$-spaces $L_{(2)}^{p, q}(X, L)$ and $L_{(2)}^{p, q}(\tilde{X}, \tilde{L})$ shown in [20]. Remember an open subset $U \subset \tilde{X}$ is called a fundamental domain of the action of the fundamental group $\pi_{1}(X)$ on $\tilde{X}$ if the following conditions are satisfied:

1. $\tilde{X}=\cup_{\gamma \in \pi_{1}(X)} \gamma(\bar{U})$;

2. $\gamma_{1}(U) \cap \gamma_{2}(U)=\varnothing$ for $\gamma_{1}, \gamma_{2} \in \pi_{1}(X), \gamma_{1} \neq \gamma_{2}$ and

3. $\bar{U} \backslash U$ has zero measure.

We construct a fundamental domain in the following way. Let $\left\{U_{k}\right\}$ be a locally finite cover of $X$ with open balls having the property that, for each $k$, there exists an open set $\tilde{U}_{k} \subset \tilde{X}$ such that $\pi: \tilde{U}_{k} \rightarrow U_{k}$ is biholomorphic with inverse $\phi_{k}: U_{k} \rightarrow \tilde{U}_{k}$. Define $W_{k}=U_{k} \backslash\left(\cup_{j<k} \bar{U}_{j} \cap U_{k}\right)$. Then, $U:=\cup_{k} \phi_{k}\left(W_{k}\right)$ is a fundamental domain.

Then, it is easy to see that

$$
L_{(2)}^{p, q}(\tilde{X}, \tilde{L}) \simeq L_{(2)} \pi_{1}(X) \otimes L_{(2)}^{p, q}(U, \tilde{L}) \simeq L_{(2)} \pi_{1}(X) \otimes L_{(2)}^{p, q}(X, L) .
$$

A basis of $L_{(2)} \pi_{1}(X)$ is formed by the functions

$$
\delta_{\gamma}\left(\gamma^{\prime}\right)= \begin{cases}1 & \text { if } \gamma=\gamma^{\prime} \\ 0 & \text { if } \gamma \neq \gamma^{\prime}\end{cases}
$$


Then, for $\alpha \in L_{(2)}^{p, q}(\tilde{X}, \tilde{L})$, the above identification is given by

$$
\alpha \simeq\left(\left.\alpha\right|_{\gamma(U)}\right)_{\gamma} \simeq \sum_{\gamma} \delta_{\gamma} \otimes \gamma^{-1} \cdot\left(\left.\alpha\right|_{\gamma(U)}\right) .
$$

Now let $\left\{\bar{\partial} \alpha_{l}\right\}$ be a sequence in $\operatorname{Im} \bar{\partial}_{1}$ that is convergent in $L_{(2)}^{p, q+1}(X, L)$. Fix a $\gamma \in$ $\pi_{1}(X)$. Then, $\left\{\bar{\partial} \tilde{\alpha}_{l}:=\delta_{\gamma} \otimes \gamma \cdot \bar{\partial} \alpha_{l}\right\}$ is a sequence in $\operatorname{Im} \bar{\partial}_{2}$ that is convergent in $L_{(2)}^{p, q+1}(\tilde{X}, \tilde{L})$. Hence, the limit is $\bar{\partial} \tilde{\alpha}$ for some $\tilde{\alpha} \in L_{(2)}^{p, q}(\tilde{X}, \tilde{L})$ due to the closeness of $\operatorname{Im} \bar{\partial}_{2}$. We define an $L^{2}$-bounded form $\alpha$ on $X$ by

$$
(\alpha, \mu)_{\omega, \varphi}:=\left(\tilde{\alpha}, \delta_{\gamma} \otimes \gamma \cdot \mu\right)_{\tilde{\omega}, \Phi}
$$

for any test form $\mu$ with proper degree on $X$. Consequently, we have

$$
\lim \left(\bar{\partial} \alpha_{l}-\bar{\partial} \alpha, \mu\right)_{\omega, \varphi}=\lim \left(\bar{\partial} \tilde{\alpha}_{l}-\bar{\partial} \tilde{\alpha}, \delta_{\gamma} \otimes \gamma \cdot \mu\right)_{\tilde{\omega}, \Phi}=0 .
$$

It exactly means that $\lim \bar{\partial} \alpha_{l}=\bar{\partial} \alpha$, hence $\operatorname{Im} \bar{\partial}_{1}$ is closed. The claim is proved. Remember the fact that $\operatorname{Im} \bar{\partial}$ is closed if and only if $\operatorname{Im} \bar{\partial}^{*}$ is closed; we have a similar conclusion between $\operatorname{Im} \bar{\partial}_{1}^{*}$ and $\operatorname{Im} \bar{\partial}_{2}^{*}$.

Due to this claim and the discussions after Corollary 2, we only need to prove that

$$
\operatorname{Im}\left(\bar{\partial}: L_{(2)}^{p, q}(\tilde{X}, \tilde{L}) \rightarrow L_{(2)}^{p, q+1}(\tilde{X}, \tilde{L})\right)
$$

and

$$
\operatorname{Im}\left(\bar{\partial}^{*}: L_{(2)}^{p, q}(\tilde{X}, \tilde{L}) \rightarrow L_{(2)}^{p, q-1}(\tilde{X}, \tilde{L})\right)
$$

are both closed with $p+q=m$. However, it is respectively equivalent to the closeness of

$$
\operatorname{Im}\left(\bar{\partial}^{*}: L_{(2)}^{p, q+2}(\tilde{X}, \tilde{L}) \rightarrow L_{(2)}^{p, q+1}(\tilde{X}, \tilde{L})\right)
$$

and

$$
\operatorname{Im}\left(\bar{\partial}: L_{(2)}^{p, q-2}(\tilde{X}, \tilde{L}) \rightarrow L_{(2)}^{p, q-1}(\tilde{X}, \tilde{L})\right),
$$

which has been verified. The proof is complete.

\section{The Injectivity Theorem}

Let $(X, \omega)$ be a weakly pseudoconvex Kähler manifold with negative sectional curvature. Let $(L, \varphi)$ be a (singular) Hermitian line bundle on $X$ such that $i \Theta_{L, \varphi} \geqslant 0$. Moreover, $\varphi$ is smooth on $Y:=X \backslash Z$ with $Z$ a closed subvariety of $X$. Firstly, we specify Hodge's theorem (Proposition 4) in this situation.

Since $X$ is weakly pseudoconvex, so will be $Y$. Then, there exists a smooth plurisubharmonic exhaustion function $\psi$ on $Y$. Set

$$
\omega_{l}=\omega+\frac{i}{l} \partial \bar{\partial} \psi^{2},
$$

which is a complete Kähler metric on $Y$ for every $l>0$. Obviously,

$$
\omega_{l_{2}} \geqslant \omega_{l_{1}} \geqslant \omega
$$

on $Y$ when $l_{1} \geqslant l_{2}>0$. Moreover, when we take large enough $l$, the sectional curvature of $\left(Y, \omega_{l}\right)$ approximates the sectional curvature of $(Y, \omega)$ and hence is negative. Therefore, $\left(Y, \omega_{l}\right)$ is a complete Kähler hyperbolic manifold by Proposition 1. 
Now, we apply Proposition 4 to $\left(Y, \omega_{l}\right)$, and obtain that

$$
\mathcal{H}_{l}^{n, q}(L) \simeq \frac{\operatorname{Ker} \bar{\partial}}{\operatorname{Im} \bar{\partial}}
$$

where

$$
\begin{aligned}
\mathcal{H}_{l}^{n, q}(L) & =\left\{\alpha \in L_{(2)}^{n, q}(Y, L) ; \bar{\partial} \alpha=0, \bar{\partial}_{l}^{*} \alpha=0\right\} \\
\operatorname{Ker} \bar{\partial} & =\operatorname{Ker}\left(\bar{\partial}: L_{(2)}^{n, q}(Y, L) \rightarrow L_{(2)}^{n, q+1}(X, L)\right),
\end{aligned}
$$

and

$$
\operatorname{Im} \bar{\partial}=\operatorname{Im}\left(\bar{\partial}: L_{(2)}^{n, q-1}(Y, L) \rightarrow L_{(2)}^{n, q}(Y, L)\right) .
$$

We use $\bar{\partial}_{l}^{*}$ to denote the adjoint operator of $\bar{\partial}$ on $Y$ defined through $\varphi$ and $\omega_{l}$. Let

$$
\begin{array}{r}
\mathcal{H}^{n, q}(L, \varphi):=\left\{\alpha \in L_{(2)}^{n, q}(X, L) ; \text { for every } l \text {, there exists an } \alpha_{l} \in \mathcal{H}_{l}^{n, q}(L)\right. \text { such that } \\
\left.\left.\alpha\right|_{Y}=\alpha_{l}+\bar{\partial} \beta_{l} \text { for some } \beta_{l} \in L_{(2)}^{n, q-1}(Y, L) \text { and } \lim \alpha_{l}=\left.\alpha\right|_{Y}\right\} .
\end{array}
$$

Under this circumstance, Hodge's theorem is formulated as

\section{Proposition 5.}

$$
\mathcal{H}^{n, q}(L, \varphi) \simeq H^{q}\left(X, K_{X} \otimes L \otimes \mathscr{I}(\varphi)\right) .
$$

Proof. Let $\alpha \in \mathcal{H}^{n, q}(L, \varphi)$. Applying Proposition 2 to each $\alpha_{l}$, we have

$$
0=\left(\partial_{\varphi}^{*} \alpha_{l}, \partial_{\varphi}^{*} \alpha_{l}\right) \omega_{l, \varphi}+\left(\left[i \Theta_{L, \varphi}, \Lambda\right] \alpha_{l}, \alpha_{l}\right)_{\omega_{l}, \varphi} .
$$

Since $i \Theta_{L, \varphi} \geqslant 0,\left(\left[i \Theta_{L, \varphi}, \Lambda\right] \alpha_{l}, \alpha_{l}\right)_{\omega_{l}, \varphi} \geqslant 0$. Therefore,

$$
\partial_{\varphi}^{*} \alpha_{l}=0,\left(\left[i \Theta_{L, \varphi}, \Lambda\right] \alpha_{l}, \alpha_{l}\right) \omega_{l, \varphi}=0
$$

In particular, $\bar{\partial} * \alpha_{l}=0$. It means that $* \alpha_{l}$ is a holomorphic $L$-valued $(n-q, 0)$-form on $Y$. On the other hand,

$$
\left\|* \alpha_{l}\right\|_{\omega_{l}, \varphi}^{2}=\left\|\alpha_{l}\right\|_{\omega_{l}, \varphi}^{2} \leqslant\left\|\left.\alpha\right|_{Y}\right\|_{\omega_{l}, \varphi}^{2} \leqslant\|\alpha\|_{\omega, \varphi}^{2}<\infty
$$

The first inequality is due to the fact that $\left.\alpha\right|_{Y}=\alpha_{l}+\bar{\partial} \beta_{l}$ and $\alpha_{l} \in \mathcal{H}_{l}^{n, q}(L)$. By canonical $L^{2}$-extension theorem [21], $* \alpha_{l}$ extends to a holomorphic $L$-valued $(n-q, 0)$-form on $X$, which is denoted by $\gamma_{l}$. Fix $l_{0}$. Then, for $l \geqslant l_{0}$,

$$
\left\|\gamma_{l}\right\|_{\omega_{l_{0}, \varphi}}^{2} \leqslant\left\|* \alpha_{l}\right\|_{\omega_{l}, \varphi}^{2} \leqslant\|\alpha\|_{\omega, \varphi}^{2}<\infty,
$$

hence $\left\{\gamma_{l}\right\}$ is uniformly bounded in $L^{2}$-norm $\|\cdot\|_{\omega_{l_{0}}, \varphi}$. Consequently, it converges to a holomorphic $L$-valued $(n-q, 0)$-form, say $\gamma$. Furthermore, as $l_{0}$ tends to $\infty$, we obtain that $\|\gamma\|_{\omega, \varphi}^{2} \leqslant\|\alpha\|_{\omega, \varphi}^{2}<\infty$. Now, it is easy to verify that

$$
\left[\omega^{q} \wedge \gamma\right] \in H^{q}\left(X, K_{X} \otimes L \otimes \mathscr{I}(\varphi)\right)
$$

We denote this morphism by $i(\alpha)=\left[\omega^{q} \wedge \gamma\right]$.

Conversely, let $[\alpha] \in H^{q}\left(X, K_{X} \otimes L \otimes \mathscr{I}(\varphi)\right)$. Let $\mathcal{L}^{q}$ be the sheaf of germs of $(n, q)$ forms $\beta$ on $X$ with values in $L$ and with measurable coefficients, such that both $|\beta|_{\omega, \varphi}^{2}$ and $|\bar{\partial} \beta|_{\omega, \varphi}^{2}$ are locally integrable. The $\bar{\partial}$ operator defines a complex of sheaves $\left(\mathcal{L}^{\bullet}, \bar{\partial}\right)$, and it is easy to verify that $\left(\mathcal{L}^{\bullet}, \bar{\partial}\right)$ is a resolution of $K_{X} \otimes L \otimes \mathscr{I}(\varphi)$. Each sheaf $\mathcal{L}^{q}$ is a $\mathcal{C}^{\infty}$-module, so $\mathcal{L}^{\bullet}$ is a resolution by acyclic sheaves. 
Then, we can find a representative $\alpha \in \Gamma\left(X, \mathcal{L}^{q}\right)$ of

$$
[\alpha] \in H^{q}\left(X, K_{X} \otimes L \otimes \mathscr{I}(\varphi)\right)
$$

through this resolution by acyclic sheaves. In other words, $\alpha$ is a $\bar{\partial}$-closed $L$-valued $(n, q)$ form on $X$ such that $|\alpha|_{\omega, \varphi}^{2}$ and $|\bar{\partial} \alpha|_{\omega, \varphi}^{2}$ are locally integrable. Moreover, through the discussions in Section 2.2, we could arrange the things so that

$$
\|\alpha\|_{\omega, \varphi}^{2}<\infty \text { and }\|\bar{\partial} \alpha\|_{\omega, \varphi}^{2}<\infty .
$$

In particular, $\left.\alpha\right|_{Y} \in L_{(2)}^{n, q}(Y, L)$. Now let $\alpha_{l}$ be the harmonic representative of $\left.\alpha\right|_{Y}$ in $L_{(2)}^{n, q}(Y, L)$. Equivalently, $\bar{\partial} \alpha_{l}=\bar{\partial}_{l}^{*} \alpha_{l}=0$. Applying the same argument of the first part, we will eventually obtain a sequence of holomorphic $L$-valued $(n-q, 0)$-forms $\left\{\gamma_{l}\right\}$ and its limit $\gamma$ on $X$. On the other hand,

$$
\left\|\alpha_{l}\right\|_{\omega_{l, \varphi}}^{2} \leqslant\left\|\left.\alpha\right|_{Y}\right\|_{\omega_{l, \varphi}}^{2} \leqslant\|\alpha\|_{\omega, \varphi}^{2}<\infty,
$$

the sequence $\left\{\alpha_{l}\right\}$ is convergent to, say $\hat{\alpha}$. Since $\omega_{l}^{q} \wedge \gamma_{l}=\alpha_{l}$,

$$
\hat{\alpha}=\lim _{l \rightarrow \infty} \alpha_{l}=\lim _{l \rightarrow \infty}\left(\omega_{l}^{q} \wedge \gamma_{l}\right)=\omega^{q} \wedge \gamma .
$$

Therefore, $\hat{\alpha} \in \mathcal{H}^{n, q}(L, \varphi)$ by definition. We denote this morphism by $j([\alpha])=\hat{\alpha}$.

It is easy to verify that $i \circ j=\operatorname{Id}$ and $j \circ i=\mathrm{Id}$. The proof is finished.

Now, we are ready to prove the injectivity theorem on a non-compact manifold. One could consult $[3,5,7,8]$ for a sophisticated comprehension for the injectivity theorem on a compact manifold.

Theorem 2 (=Theorem 1$)$. Let $(X, \omega)$ be a weakly pseudoconvex Kähler manifold such that

$$
\sec \leqslant-K
$$

for some positive constant $K$. Let $\left(L, \varphi_{L}\right)$ and $\left(H, \varphi_{H}\right)$ be two (singular) Hermitian line bundles on $X$. Assume the following conditions:

1. There exists a closed subvariety $Z$ on $X$ such that $\varphi_{L}$ and $\varphi_{H}$ are both smooth on $X \backslash Z$;

2. $i \Theta_{L, \varphi_{L}} \geqslant 0$ and $i \Theta_{H, \varphi_{H}} \geqslant 0$ on $X$;

3. $i \Theta_{L, \varphi_{L}} \geqslant \delta i \Theta_{H, \varphi_{H}}$ for some positive number $\delta$.

For a (non-zero) section sof $H$ with $\sup _{X}|s|^{2} e^{-\varphi_{H}}<\infty$, the multiplication map induced by the tensor product with $s$

$$
\Phi: H^{q}\left(X, K_{X} \otimes L \otimes \mathscr{I}\left(\varphi_{L}\right)\right) \rightarrow H^{q}\left(X, K_{X} \otimes L \otimes H \otimes \mathscr{I}\left(\varphi_{L}+\varphi_{H}\right)\right)
$$

is (well-defined and) injective for any $q \geqslant 0$.

Proof. By Proposition 5, it is enough to prove that

$$
\otimes s: \mathcal{H}^{n, q}\left(L, \varphi_{L}\right) \rightarrow \mathcal{H}^{n, q}\left(L \otimes H, \varphi_{L}+\varphi_{H}\right)
$$

is well-defined, hence injective. In other words, let $\alpha \in \mathcal{H}^{n, q}\left(L, \varphi_{L}\right)$, and we should prove that $s \alpha \in \mathcal{H}^{n, q}\left(L \otimes H, \varphi_{L}+\varphi_{H}\right)$.

In fact, since $\alpha \in \mathcal{H}^{n, q}\left(L, \varphi_{L}\right)$, there exists $\alpha_{l} \in \mathcal{H}_{l}^{n, q}(L)$ and

$$
\beta_{l} \in L_{(2)}^{n, q-1}(Y, L)
$$


with $\alpha=\alpha_{l}+\bar{\partial} \beta_{l}$. Applying Proposition 2, we obtain that

$$
0=\left(\partial_{\varphi_{L}}^{*} \alpha_{l}, \partial_{\varphi_{L}}^{*} \alpha_{l}\right)_{\omega_{l}, \varphi_{L}}+\left(\left[i \Theta_{L, \varphi_{L}}, \Lambda\right] \alpha_{l}, \alpha_{l}\right)_{\omega_{l}, \varphi_{L}} .
$$

Notice that $i \Theta_{L, \varphi_{L}} \geqslant 0,\left(\left[i \Theta_{L, \varphi_{L}}, \Lambda\right] \alpha_{l}, \alpha_{l}\right)_{\omega_{l}, \varphi_{L}} \geqslant 0$. Hence,

$$
\left(\partial_{\varphi_{L}}^{*} \alpha_{l}, \partial_{\varphi_{L}}^{*} \alpha_{l}\right)_{\omega_{l}, \varphi_{L}}=\left(\left[i \Theta_{L, \varphi_{L}}, \Lambda\right] \alpha_{l}, \alpha_{l}\right)_{\omega_{l}, \varphi_{L}}=0 .
$$

In particular, $\partial_{\varphi_{L}}^{*} \alpha_{l}=0$.

Now, apply Proposition 2 again on $s \alpha_{l}$ and observe that $\bar{\partial}\left(s \alpha_{l}\right)=0$, we obtain that

$$
\begin{aligned}
0 & \leqslant\left(\bar{\partial}_{l}^{*}\left(s \alpha_{l}\right), \bar{\partial}_{l}^{*}\left(s \alpha_{l}\right)\right)_{\omega_{l}, \varphi_{L}+\varphi_{H}} \\
& =\left(\partial_{\varphi_{L}+\varphi_{H}}^{*}\left(s \alpha_{l}\right), \partial_{\varphi_{L}+\varphi_{H}}^{*}\left(s \alpha_{l}\right)\right)_{\omega_{l}, \varphi_{L}+\varphi_{H}}+\left(\left[i \Theta_{L \otimes H, \varphi_{L}+\varphi_{H}}, \Lambda\right]\left(s \alpha_{l}\right), s \alpha_{l}\right) \omega_{l, \varphi_{L}+\varphi_{H}} .
\end{aligned}
$$

Since $\partial_{\varphi_{L}+\varphi_{H}}^{*}\left(s \alpha_{l}\right)=s \partial_{\varphi_{L}}^{*} \alpha_{l}=0$, and

$$
\begin{aligned}
0 & \leqslant\left(\left[i \Theta_{L \otimes H, \varphi_{L}+\varphi_{H}}, \Lambda\right]\left(s \alpha_{l}\right), s \alpha_{l}\right) \omega_{l}, \varphi_{L}+\varphi_{H} \\
& \leqslant \sup _{X}|s|^{2} e^{-\varphi_{H}}\left(\left[i \Theta_{L \otimes H, \varphi_{L}+\varphi_{H}}, \Lambda\right] \alpha_{l}, \alpha_{l}\right) \omega_{l}, \varphi_{L} \\
& \leqslant\left(1+\frac{1}{\delta}\right) \sup _{X}|s|^{2} e^{-\varphi_{H}}\left(\left[i \Theta_{L, \varphi_{L}}, \Lambda\right] \alpha_{l}, \alpha_{l}\right)_{\omega_{l}, \varphi_{L}} \\
& =0,
\end{aligned}
$$

it is easy to see that

$$
\left(\bar{\partial}_{l}^{*}\left(s \alpha_{l}\right), \bar{\partial}_{l}^{*}\left(s \alpha_{l}\right)\right)_{\omega_{l}, \varphi_{L}+\varphi_{H}}=0 .
$$

In particular, $\bar{\partial}_{l}^{*}\left(s \alpha_{l}\right)=0$, hence $s \alpha_{l} \in \mathcal{H}_{l}^{n, q}(L \otimes H)$. Obviously, $s \alpha=s \alpha_{l}+\bar{\partial}\left(s \beta_{l}\right)$ and $s \beta_{l} \in L_{(2)}^{n, q-1}(Y, L \otimes H)$. Therefore,

$$
s \alpha \in \mathcal{H}^{n, q}\left(L \otimes H, \varphi_{L}+\varphi_{H}\right)
$$

by definition. The injectivity is now obvious.

Proof of Corollary 1. Consider the short exact sequence

$$
\begin{gathered}
0 \rightarrow K_{X} \otimes L \otimes \mathscr{I}\left(\varphi_{L}+\varphi\right) \rightarrow K_{X} \otimes L \otimes \mathscr{I}\left(\varphi_{L}\right) \rightarrow \\
K_{X} \otimes L \otimes \mathscr{I}\left(\varphi_{L}\right) / \mathscr{I}\left(\varphi_{L}+\varphi\right) \rightarrow 0 .
\end{gathered}
$$

The associated cohomology long exact sequence implies that the surjectivity of

$$
H^{0}\left(X, K_{X} \otimes L \otimes \mathscr{I}\left(\varphi_{L}\right)\right) \rightarrow H^{0}\left(X, K_{X} \otimes L \otimes \mathscr{I}\left(\varphi_{L}\right) / \mathscr{I}\left(\varphi_{L}+\varphi\right)\right)
$$

is equivalent to the injectivity of

$$
H^{1}\left(X, K_{X} \otimes L \otimes \mathscr{I}\left(\varphi_{L}+\varphi\right)\right) \rightarrow H^{1}\left(X, K_{X} \otimes L \otimes \mathscr{I}\left(\varphi_{L}\right)\right) .
$$

Applying Proposition 5, it reduces to prove that

$$
\begin{aligned}
\mathcal{H}^{n, 1}\left(L, \varphi_{L}+\varphi\right) & \rightarrow \mathcal{H}^{n, 1}\left(L, \varphi_{L}\right) \\
\alpha & \mapsto \alpha
\end{aligned}
$$

is well-defined.

In fact, let $\alpha_{l} \in \mathcal{H}_{l}^{n, 1}\left(L, \varphi_{L}+\varphi\right)$ and $\beta_{l} \in L_{(2)}^{n, 0}(Y, L)_{\varphi_{L}+\varphi}$ such that

$$
\alpha=\alpha_{l}+\bar{\partial} \beta_{l} .
$$


Obviously, $\beta_{l} \in L_{(2)}^{n, 0}(Y, L)_{\varphi_{L}}$. Here, $\mathcal{H}_{l}^{n, 1}\left(L, \varphi_{L}+\varphi\right), L_{(2)}^{n, 0}(Y, L)_{\varphi_{L}+\varphi}$ and $L_{(2)}^{n, 0}(Y, L)_{\varphi_{L}}$ are understood in an obvious way. Moreover, applying Proposition 2 with $\varphi_{L}+\varphi$, we obtain that

$$
\partial_{\varphi_{L}+\varphi}^{*} \alpha_{l}=0 \text { and }\left(\left[i \Theta_{L, \varphi_{L}+\varphi}, \Lambda\right] \alpha_{l}, \alpha_{l}\right)_{\omega_{l}, \varphi_{L}+\varphi}=0 .
$$

Applying Proposition 2 one more time with $\varphi_{L}$, then

$$
\begin{aligned}
0 & \leqslant\left(\bar{\partial}_{\varphi_{L}}^{*} \alpha_{l}, \bar{\partial}_{\varphi_{L}}^{*} \alpha_{l}\right)_{\omega_{l}, \varphi_{L}} \\
& =\left(\partial_{\varphi_{L}}^{*} \alpha_{l}, \partial_{\varphi_{L}}^{*} \alpha_{l}\right)_{\omega_{l}, \varphi_{L}}+\left(\left[i \Theta_{L, \varphi_{L}}, \Lambda\right] \alpha_{l}, \alpha_{l}\right)_{\omega_{l}, \varphi_{L}} .
\end{aligned}
$$

Since $\partial_{\varphi_{L}+\varphi}^{*}=(-1)^{n(n+2)+1} * \bar{\partial} *=\partial_{\varphi_{L}}^{*}$, we also have $\partial_{\varphi_{L}}^{*} \alpha_{l}=0$. On the other hand,

$$
\begin{aligned}
0 & \leqslant\left(\left[i \Theta_{L, \varphi_{L}}, \Lambda\right] \alpha_{l}, \alpha_{l}\right) \omega_{l, \varphi_{L}} \\
& \leqslant\left(\left[i \Theta_{L, \varphi_{L}}, \Lambda\right] \alpha_{l}, \alpha_{l}\right)_{\omega_{l}, \varphi_{L}+\varphi} \\
& \leqslant\left(1+\frac{1}{\delta}\right)\left(\left[i \Theta_{L, \varphi_{L}+\varphi}, \Lambda\right] \alpha_{l}, \alpha_{l}\right) \omega_{l, \varphi_{L}+\varphi} \\
& =0
\end{aligned}
$$

The last inequality comes from the assumption that for $\delta \in(0, \varepsilon)$,

$$
i \Theta_{L, \varphi_{L}}+(1+\delta) i \partial \bar{\partial} \varphi \geqslant 0 .
$$

In summary, $\bar{\partial}_{\varphi_{L}}^{*} \alpha_{l}=0$. Therefore, $\alpha_{l} \in \mathcal{H}_{l}^{n, 1}\left(L, \varphi_{L}\right) . \alpha \in \mathcal{H}^{n, 1}\left(L, \varphi_{L}\right)$ by definition. The proof is complete.

\section{Conclusions}

We establish an injectivity theorem on a weakly pseudoconvex Kähler manifold $X$ with negative sectional curvature. In particular, $X$ is not necessary to be compact. As an application, we obtain an $L^{2}$-extension theorem concerning the subvariety that is not necessary to be reduced. Such type of extension theorem is of crucial importance in complex geometry.

Funding: This research was funded by China Postdoctoral Science Foundation Grant No. 2019M661328.

Acknowledgments: The author thanks the referees for detailed and constructive criticism of the original manuscript.

Conflicts of Interest: The author declares no conflict of interest.

\section{References}

1. Kollár, J. Higher direct images of dualizing sheaves I. Ann. Math. 1986, 123, 11-42. [CrossRef]

2. Kollár, J. Higher direct images of dualizing sheaves II. Ann. Math. 1986, 124, 171-202. [CrossRef]

3. Ambro, F. An injectivity theorem. Compos. Math. 2014, 150, 999-1023. [CrossRef]

4. Enoki, I. Kawamata-Viehweg vanishing theorem for compact Kähler manifolds. In Einstein Metrics and Yang-Mills Connections; CRC Press: Boca Raton, FL, USA, 1993; pp. 59-68.

5. Fujino, O. A transcendental approach to Kollár's injectivity theorem. Osaka J. Math. 2012, 49, 833-852. [CrossRef]

6. Fujino, O.; Matsumura, S. Injectivity theorem for pseudo-effective line bundles and its applications. arXiv 2016, arXiv:1605.02284.

7. Gongyo, Y.; Matsumura, S. Versions of injectivity and extension theorems. In Annales Scientifiques de l'Ecole Normale Superieure; Societe Mathematique de France: Paris, France, 2017; Volume 50, pp. 479-502.

8. Matsumura, S. A Nadel vanishing theorem via injective theorems. Math. Ann. 2014, 359, 785-802. [CrossRef]

9. Matsumura, S. Injectivity theorems with multiplier ideal sheaves and their applications. Complex Anal. Geom. 2015, 144, 241-255.

10. Cao, J.; Demailly, J.-P.; Matsumura, S. A general extension theorem for cohomology classes on non reduced analytic subspaces. Sci. China Math. 2017, 60, 949-962. [CrossRef]

11. Zhou, X.; Zhu, R. Extension of cohomology classed and holomorphic sections defined on subvarieties. arXiv 2019, arXiv:1909.08822.

12. Demailly, J.-P.; Peternell, T.; Schneider, M. Pseudo-effective line bundles on compact Kähler manifolds. Int. J. Math. 2001, 6, 689-741. [CrossRef] 
13. Gromov, M. Kähler hyperbolicity and $L_{2}$-Hodge theory. J. Differ. Geom. 1991, 33, 263-292. [CrossRef]

14. Griffiths, P.; Harris, J. Principles of Algebraic Geometry; Wiley: New York, NY, USA, 1978.

15. Morrow, J.; Kodaira, K. Complex Manifolds; Holt, Rinehart and Winston: New York, USA, 1971.

16. Demailly, J.-P. Analytic Methods in Algebraic Geometry; International Press: Somerville, MA, USA, 2012.

17. Nadel, A.M. Multiplier ideal sheaves and Kähler-Einstein metrics of positive scalar curvature. Ann. Math. 1990, 132, 549-596. [CrossRef]

18. Chen, B.; Yang, X. Compact Kähler manifolds homotopic to negatively curved Riemannian manifolds. Math. Ann. 2018, 370, 1477-1489. [CrossRef]

19. Huang, T. $L^{2}$ vanishing theorem on some Kähler manifolds. Isr. J. Math. 2021, 241, 147-186. [CrossRef]

20. Ma, X.; Marinescu, G. Holomorphic Morse Inequalities and Bergman Kernels; Progress in Mathematics; Springer: Berlin/Heidelberg, Germany, 2007; Volume 254.

21. Ohsawa, T. Analysis of Several Complex Variables; American Mathematical Soc.: Providence, RI, USA, 2002. 